\title{
Realizations of Symmetric Maps by Symmetric Polyhedra*
}

\author{
Branko Grünbaum \\ University of Washington, Box 354350 , \\ Seattle, WA 98195-4350, USA \\ grunbaum@math.washington.edu
}

Dedicated to Jörg M. Wills on his sixtieth birthday

\begin{abstract}
Representations by polyhedra of several regular maps of positive genus are developed. The groups of symmetries of some of these polyhedra are proper subgroups of their groups of combinatorial symmetries, but we show that faithful representations can be obtained if appropriately general polyhedra are admitted. The general definition of polyhedra, which may be useful in other contexts as well, is also discussed, as are some of the reasons for its absence from the literature.
\end{abstract}

\section{Introduction, Definitions, and an Illustration}

Representations of maps by polyhedra have attracted considerable attention in recent years; see, for example, the surveys [20], [2], and the references given there. In particular, there has been great interest in maps with a high degree of symmetry, and in realizations of such maps by polyhedra with a high degree of symmetry; the references [1], [2], [14], [16]-[18], and [22]-[28] show how important was the rôle played by Wills in these developments. It is the aim of this short note to show by a few examples how the range of possibilities can be greatly extended if one eliminates certain restrictions on polyhedra. These restrictions arose by tradition, in part through errors and misunderstandings, and have no justification in the context of a general theory of polyhedra.

In order to avoid lengthy introductions, here are quick definitions of the concepts we need. For us (compare, for example, [6], [9], [26], [27], and [30]), a map or cell complex is a cell-decomposition of a compact 2-manifold $\mathcal{M}$ without boundary. Each cell $C$ of $\mathcal{M}$ is a closed topological disk (a face of $\mathcal{M}$ ), with boundary bd $C$ which is

\footnotetext{
* This research was supported in part by NSF Grant DMS-9300657.
} 
homeomorphic to a circle and is endowed with a partition into a finite number of arcs (the edges of $\mathcal{M}$ ) by points (the vertices of $\mathcal{M}$ ) belonging to three or more cells.

Departing from the usual definitions, we say that a family $\mathcal{V}$ of points and a family $\mathcal{E}$ of segments in Euclidean 3 -space $\mathbb{E}^{3}$ constitute a polyhedron $P$ realizing (or representing) a map $\mathcal{M}$ provided:

(i) There is a surjection of the set of vertices of $\mathcal{M}$ onto $\mathcal{V}$; the image-point of each vertex of $\mathcal{M}$ is labeled by the same label as the vertex itself, and is called a vertex of $P$.

(ii) There is a surjection of the set of edges of $\mathcal{M}$ onto $\mathcal{E}$; the image of each edge of $\mathcal{M}$ is called an edge of $P$. This surjection is induced by the one in (i), that is, incidences of vertices and edges in $\mathcal{M}$ are preserved in their images.

(iii) The images of the edges of each face of $\mathcal{M}$ are coplanar. The resulting planar circuit of vertices and edges of $P$ is called a face of $P$.

The above definition does not preclude the possibility of distinct vertices of $\mathcal{M}$ being mapped onto the same point of $\mathbb{E}^{3}$. By abuse of language we say that such vertices coincide, although, even if a point represents two or more vertices, these vertices are still distinct and distinguishable since each has its own label. Naturally, this entails the possibility that an edge may be represented by a segment with coinciding endpoints, or that a face may appear to have too few edges - but the combinatorial structure is still preserved by the labels carried by the vertices. An illustration of these concepts by the example in Fig. 1 should ease their assimilation and show one aspect of their usefulness.

The different parts of Fig. 1 show several realizations of the map of the truncated tetrahedron (3.6.6), consisting of four triangular and four hexagonal cells. The uniform polyhedron shown in (a) is modified in a continuous way while keeping it isogonal (that is, with symmetries acting transitively on the vertices), and several stages of the modification are shown in a sequence indicated by the arrows. The labels of the vertices are shown in several diagrams; in other parts they can be inferred and are omitted to reduce clutter. In each polyhedron one triangle and one hexagon are highlighted by heavy lines. In (c) the triplets of vertices that determine triangles are represented by the same point; their identities are preserved by the labels, and they reemerge as distinct points in (d). The hexagons in (c) have three pairs of coinciding vertices each; in (d) the hexagons have distinct vertices, but now are self-intersecting. In (d), (e), and (f) the hexagons are of three different topological types. At stage (h) triplets of vertices again coincide, but in a different way than in (c). Now triangles are equilateral, and hexagons are regular as well, of type $\left\{\frac{6}{2}\right\}$. (More about such regular polygons in Section 5 below.) Since it is an isogonal polyhedron with regular polygons as faces, the polyhedron shown in (h) is a uniform polyhedron and may be characterized by the symbol $\left(3 \cdot \frac{6}{2} \cdot \frac{6}{2}\right)$. Continuing the sequence, hexagons in (j), (k), (l) again pass through three topological types, reaching in $(\mathrm{m})$ a stage in which each hexagon has three pairs of coinciding vertices-distinct from the coinciding pairs in (c). The illustration ends in (o) where the uniform (3.6.6) is reached. This is the reflection in the origin of the polyhedron in (a); hence the sequence could be continued through the same steps as from (a) to close a circuit of isogonal representations of the same map.

It should be noted that the group of combinatorial symmetries of the map (3.6.6) is isomorphic to the group of isometric symmetries of all the polyhedra shown in Fig. 1. 

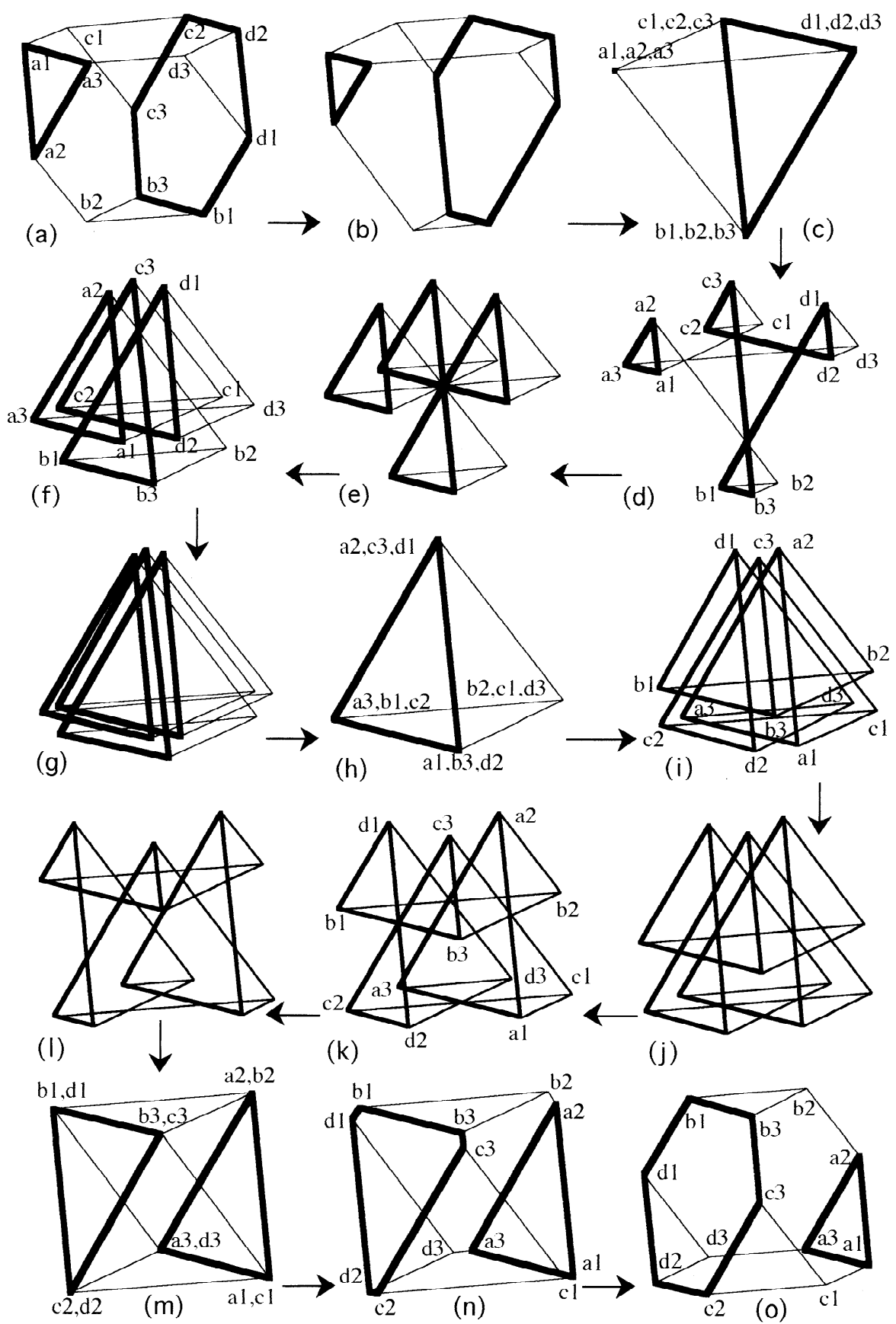

(j)

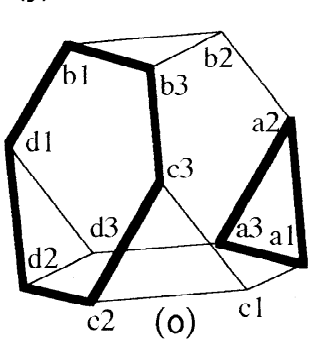

Fig. 1 


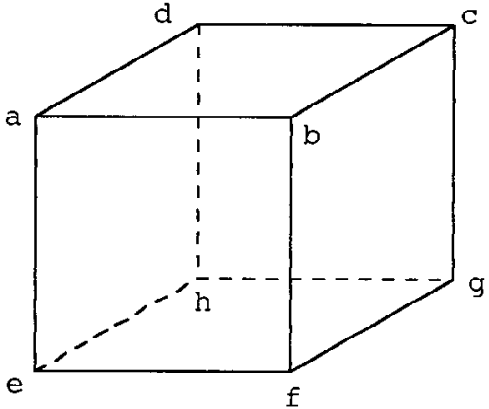

(a)

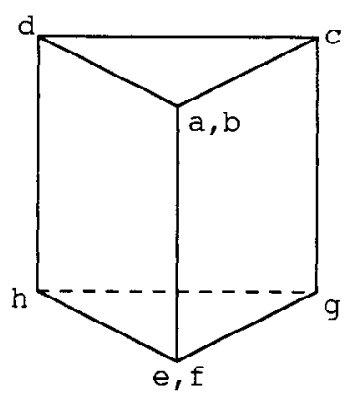

(b)

Fig. 2

Extending the terminology of Wills [26], [27], we could say that these polyhedra are realizations of index 1 of the underlying map. Naturally, while all the polyhedra in Fig. 1 are mutually equivalent combinatorially and with respect to symmetries, they are geometrically distinct. In fact, in any sensible geometric classification of polyhedra, they should all be considered as having different types, except that (f) and (g), and (i) and (j) should be assigned to the same type. The tentative form of the last statement stems from the fact that so far no sensible geometric classification of general polyhedra has been proposed; for certain restricted classes see, for example, [6], [8], and [9].

One circumstance concerning symmetries should be mentioned. The map of the cube shown in Fig. 2(a) has also the realization shown in Fig. 2(b), which appears to be an Archimedean three-sided prism. Although that prism has a $120^{\circ}$ rotational symmetry, this is not a symmetry of the polyhedron actually represented in Fig. 2(b), since this rotation is not compatible with the combinatorial structure of the polyhedron. The polyhedron in (b) has as nontrivial symmetries only the reflections in the planes that are perpendicular bisectors of the edges $c d$ or $a e$, and the half-turn which is their product. In what follows, all symmetries of polyhedra will be understood with the interpretation that the isometry has to be compatible with an incidence-preserving permutation of the labels.

Now we are ready for some more interesting examples.

\section{Examples of Regular Polyhedra Representing Regular Maps}

We start by considering a regular map, sometimes denoted by $\{4,6 \mid 5\}$, dual to the map $\left\{\begin{array}{l}6 \\ 6\end{array}\right\}_{2,0}$ described by Sherk [21]; it is orientable of genus 5, with 16 vertices of valence 6, 24 quadrangular faces, and 48 edges, and is map \#48.51 in Wilson's catalog [29] of regular maps. The map is illustrated in Fig. 3.

A realization of this map by an isohedral polyhedron (that is, a polyhedron in which all faces are equivalent under symmetries) was found by Schulte and Wills [17], [19]; the faces are congruent trapezes. In the terminology of Schulte and Wills [17], this is a Kepler-Poinsot-type polyhedron, in other words, a polyhedron in which faces are polygonal disks which may intersect and cross other faces, and with the symmetry group of a Platonic solid. (In [17] Kepler-Poinsot-type polyhedra are required to satisfy some 


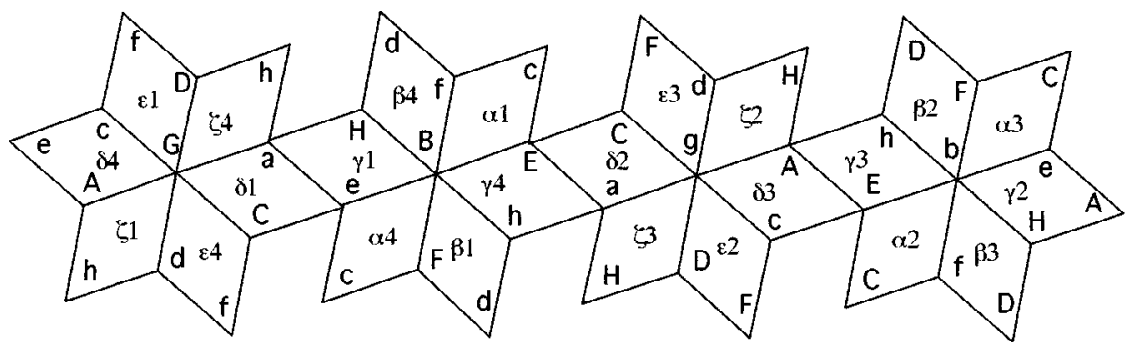

Fig. 3

additional conditions, which we do not wish to impose.) We construct this polyhedron in the following way. We start with a cube, and replace each of its square faces by four trapezes; the long side of each trapeze is one of the sides of the original square, while the opposite side is obtained from the opposite side of the square by shrinking it in a fixed ratio $r$ (with $0<r<1$ ) toward the center of the cube. (Following our definition of polyhedra, we consider the trapezes in question only as polygonal lines.) We may note that there are pairs of vertices which are incident with pairs of faces without defining an edge; however, this has no relevance to our discussion-and, moreover, it occurs even among the traditional Kepler-Poinsot regular polyhedra. (The explanation of the construction in [17] and [19] is slightly different, but yields the same result; the present explanation is more convenient for modifications we shall discuss later.) Two views of the polyhedron resulting for $r=\frac{2}{3}$ are given in Fig. 4; in Fig. 4(a) one face is emphasized, and the other faces can be read-off from Fig. 3. Fig. 4(b) shows a view in which some of the faces have been rendered as polygonal disks, in order to illustrate the intersections of the various faces.

Varying the ratio $r$ yields distinct (nonsimilar) realization of the map $\{4,6 \mid 5\}$. The (isometric) symmetries of each of these polyhedra form a subgroup of index 4 in the group of (combinatorial) symmetries of the map. However, an interesting change occurs

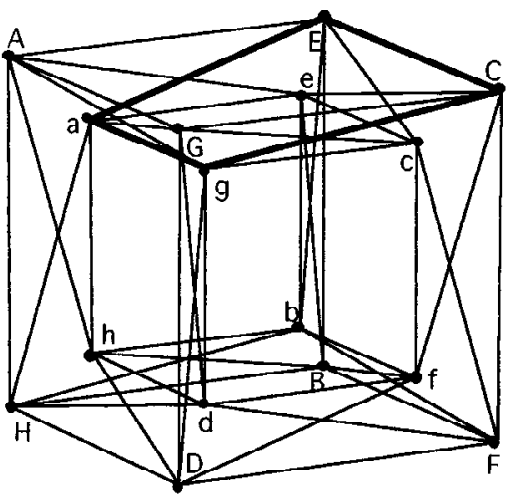

(a)

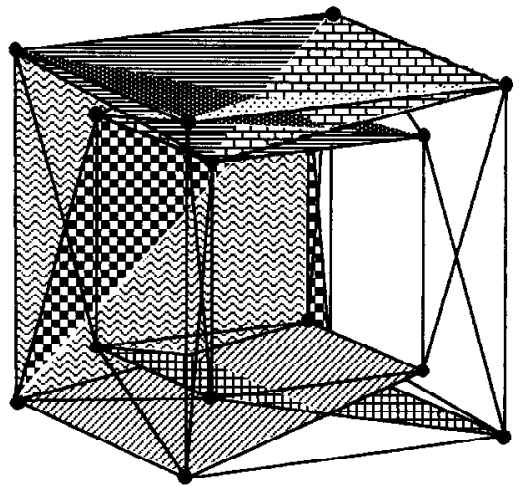

(b)

Fig. 4 


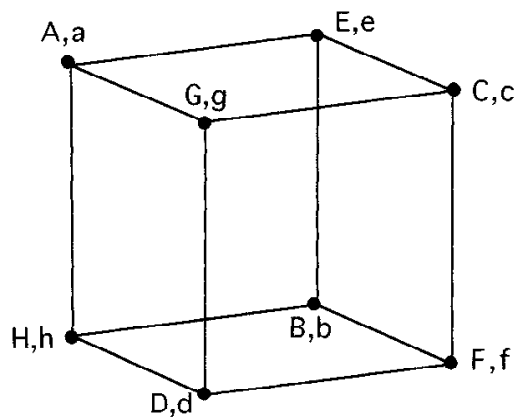

Fig. 5

if we let $r \rightarrow 1$. Then we obtain, in the limit $r=1$, a polyhedron $R$ which is regular and has all the symmetries of the map $\{4,6 \mid 5\}$. This polyhedron $R$, shown in Fig. 5, has the Schläfli symbol $\left\{4, \frac{6}{2}\right\}$. It looks like a cube, but in fact $R$ is a realization of $\{4,6 \mid 5\}$ in which each vertex of the cube corresponds to two vertices of $R$ as indicated by the labels, and each face of the cube represents four faces of the map.

We note in passing that allowing $r$ to have negative values leads to realizations with index 4 of the same map $\{4,6 \mid 5\}$ by polyhedra in which the faces are self-intersecting quadrangles. This is illustrated for $r=-\frac{2}{3}$ in Fig. 6(a), where one of the faces is emphasized. For $r=-1$ we again have special circumstances; the resulting polyhedron, shown in Fig. 6(b), is a realization of index 2 of the map.

It is of some interest to consider the map dual to $\{4,6 \mid 5\}$ (which is denoted $\left\{\begin{array}{l}6 \\ 6\end{array}\right\}_{2,0}$ by Sherk [21] and is map \#48.49 in [29]), and its realization by the polars of the polyhedra discussed above. (A different realization of this map by a Kepler-Poinsot-type polyhedron of index 4 is described by Wills [25].) The map is shown in Fig. 7, and a polar of

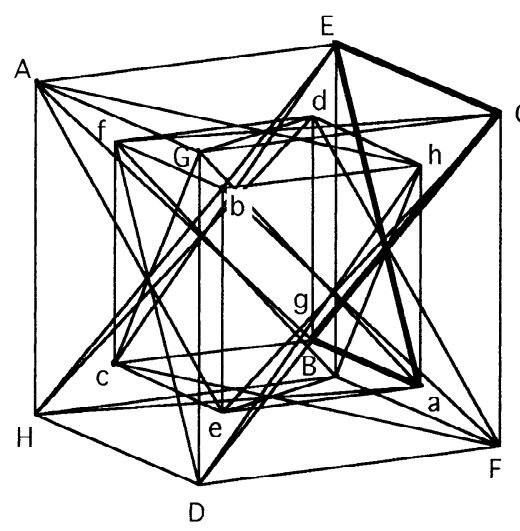

(a)

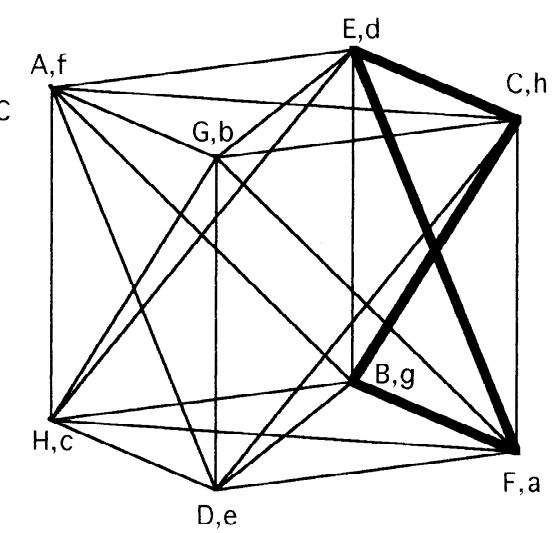

(b)

Fig. 6 


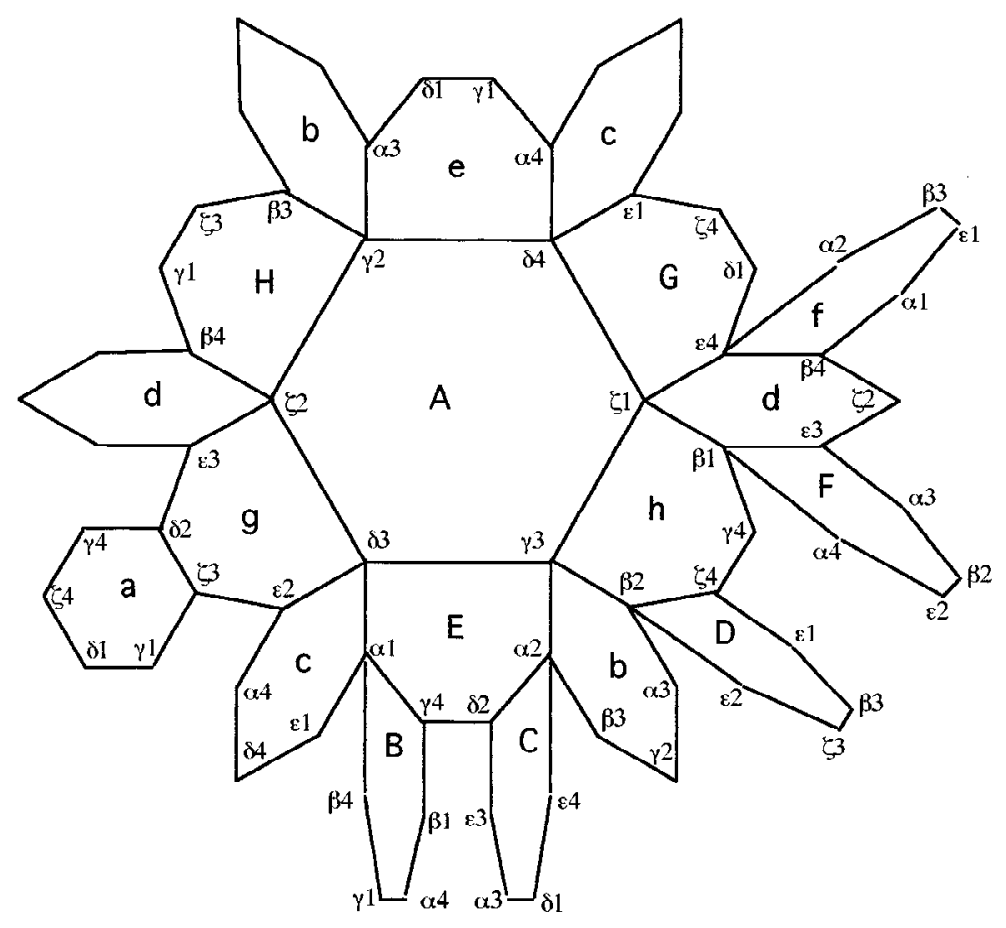

Fig. 7

the polyhedron in Fig. 4 is shown in Fig. 8. Two of its hexagonal faces, contained in parallel planes, are shown by heavy lines of different shades. The polyhedron in Fig. 8 is isogonal and its hexagonal faces all have rotation number 2 . The ratio $r$ represents here the ratio of distances of parallel faces from the center. As $r \rightarrow 1$, the faces become regular polygons $\left\{\frac{6}{2}\right\}$, and the limit polyhedron $R$ (shown in Fig. 9) is again regular, with symmetry group isomorphic to the group of combinatorial symmetries of the map. It is polar to the polyhedron $R^{*}$ in Fig. 5, and its Schläfli symbol is $\left\{\frac{6}{2}, 4\right\}$.

\section{Some Less-Than-Regular Examples}

We next consider a slightly different dual pair of maps. The toroidal map in Fig. 10 is isohedral and isotoxal (the edges form one orbit under combinatorial symmetries of the map), but is not regular. It can be realized by a family of isohedral but not isotoxal polyhedra, one representative of which is shown in Fig. 11. Polyhedra of this kind are obtained by starting from a cube, selecting four nonadjacent vertices (which correspond to E, F, G, H in Fig. 11(a)) and leaving them unchanged, while the other four vertices are replaced by pairs of vertices. One vertex in each pair is closer to the center by a factor $r$, the other is farther away to such an extent that the quadrangles of interest remain planar. In general, this is a representation whose symmetry group has index 2 as a subgroup of 


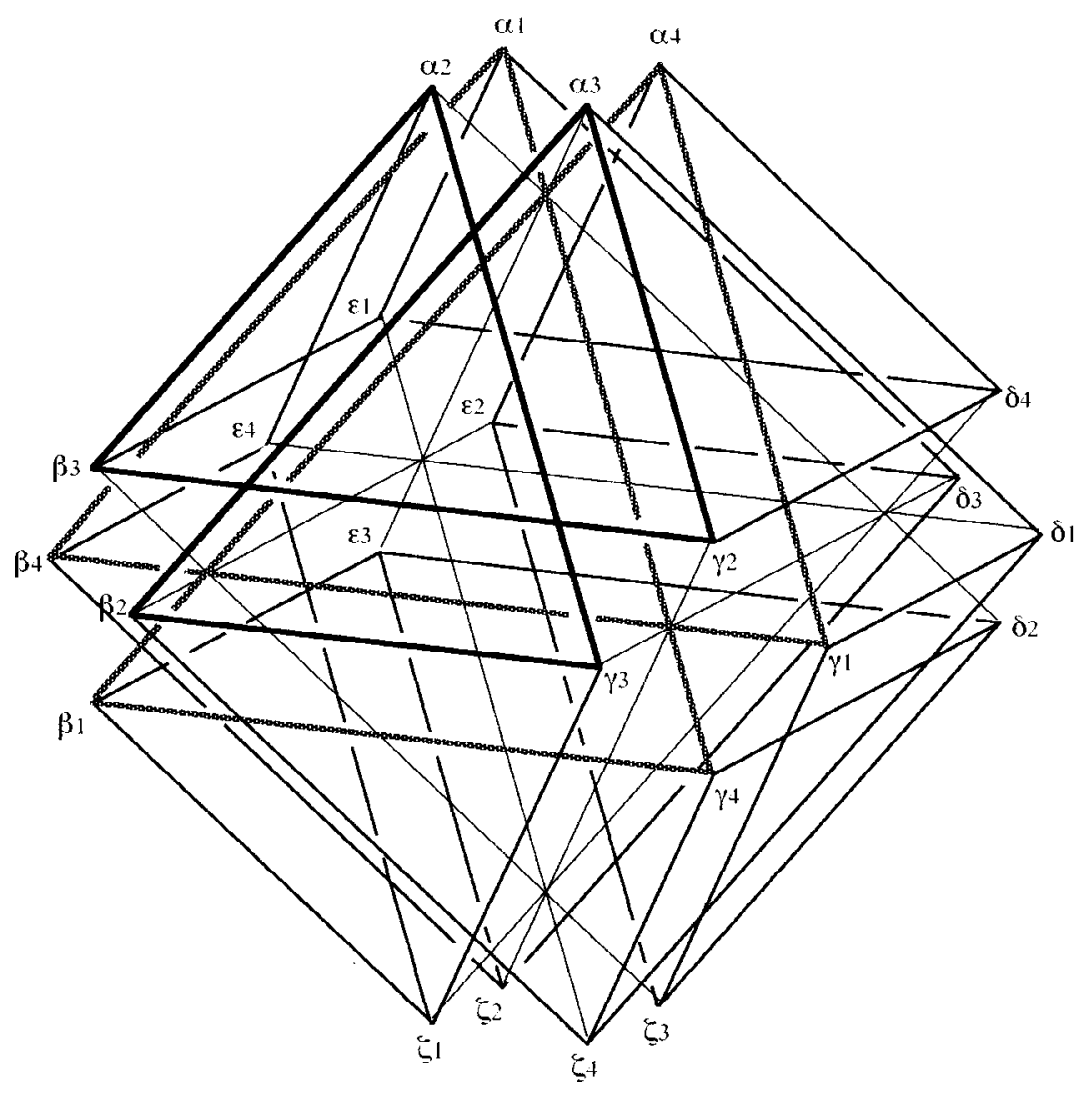

Fig. 8

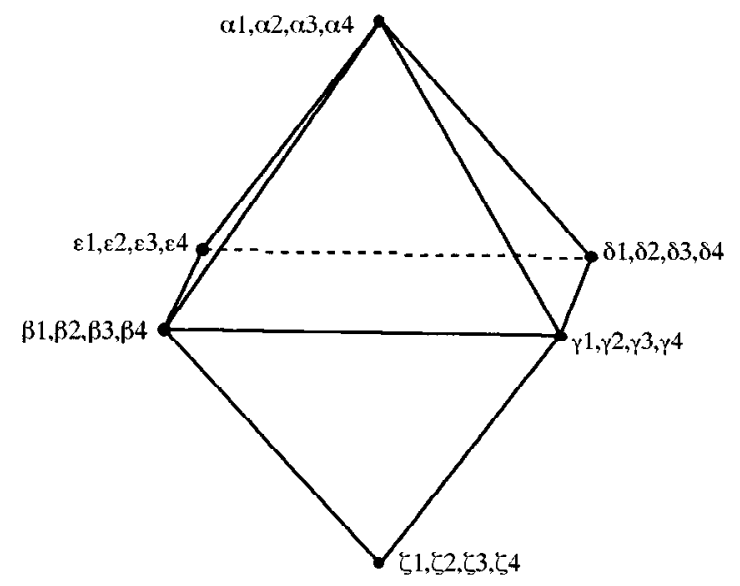

Fig. 9 


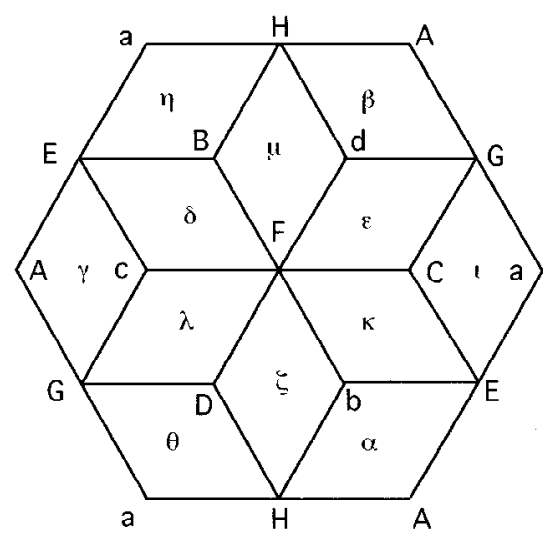

Fig. 10

the group of combinatorial symmetries of the map in Fig. 10. However, in the special case $r=1$ (shown in Fig. 12) we again have additional symmetries and the polyhedron has a symmetry group isomorphic to the group of automorphisms of the map. Moreover, in this special case the faces are squares, and the polyhedron is isohedral and isotoxal, but it is not isogonal since some of its vertices are 3-valent while other vertices have valence 6 . This is a situation which seems not to have been observed before.

The toroidal map in Fig. 13 is isogonal and isotoxal but not isohedral; it is dual to the map in Fig. 10. Polyhedra of the kind shown in Fig. 14 are isogonal but not isotoxal realizations of index 2 of this map. In general, its faces are four pairs of equilateral triangles, the members of each pair in parallel but distinct planes, and four hexagons of rotation number 2, in planes parallel to the planes of the triangles. In the special case shown in Fig. 15, the planes of the triangles of each pair coincide, the triangles themselves are congruent, and the hexagons are regular of type $\left\{\frac{6}{2}\right\}$. Thus the polyhedron in Fig. 15 is a uniform polyhedron, with symbol $\left(3 \cdot \frac{6}{2} \cdot 3 \cdot \frac{6}{2}\right)$.

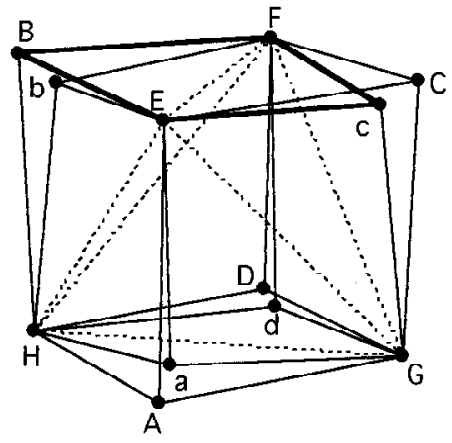

(a)

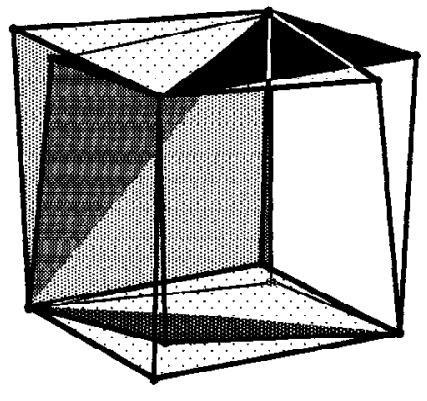

(b)

Fig. 11 


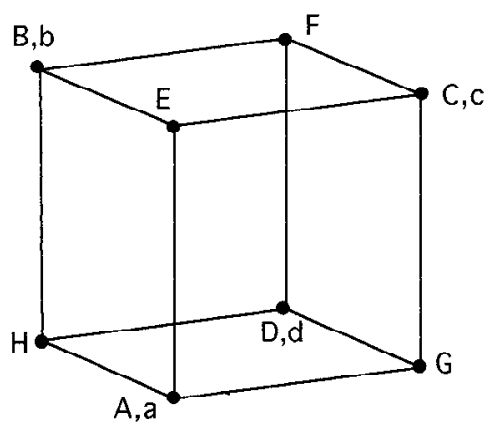

Fig. 12

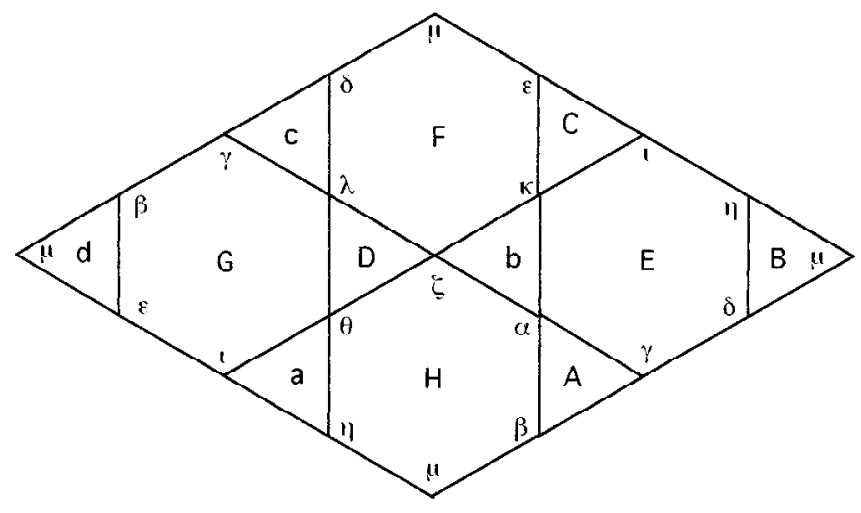

Fig. 13

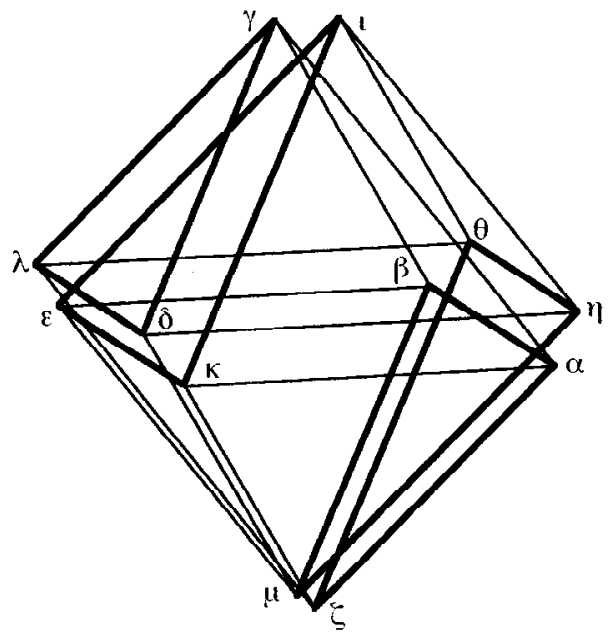

Fig. 14 


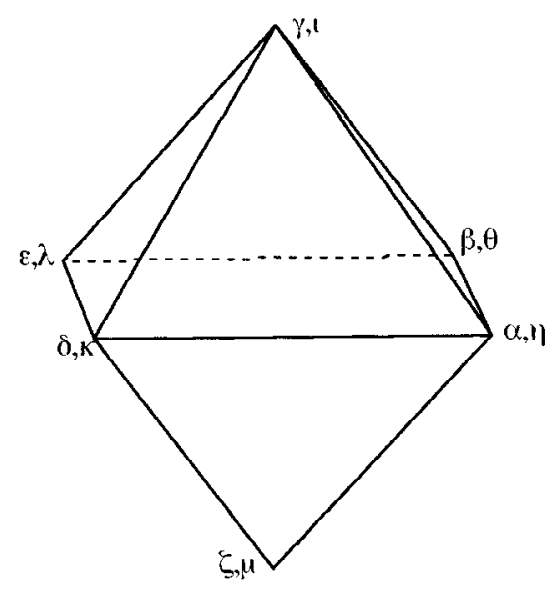

Fig. 15

The polyhedron $\left(3 \cdot \frac{6}{2} \cdot 3 \cdot \frac{6}{2}\right)$ in Fig. 15 can also be described as resulting from an octahedron in which half of the triangles have been replaced by regular hexagons of type $\left\{\frac{6}{2}\right\}$, while the remaining triangles have been replaced by pairs of triangles. Clearly, similar changes in several other uniform polyhedra lead to additional "new" uniform polyhedra, such as $\left(3 \cdot \frac{10}{2} \cdot 3 \cdot \frac{10}{2}\right),\left(\frac{6}{2} \cdot 5 \cdot \frac{6}{2} \cdot 5\right),\left(5 \cdot \frac{10}{4} \cdot 5 \cdot \frac{10}{4}\right)$, etc.

\section{A Highly Symmetric Slab}

As the final example of the application of the same ideas we start with the regular tiling of the plane by hexagons, illustrated in Fig. 16. We replace each hexagon by a set of six hexagons, according to the following procedure; the hexagonal tiling is taken to be horizontal in $\mathbb{E}^{3}$. Let $z$ be a fixed real number; any two adjacent vertices of a hexagonal tile are moved vertically upward through distance $z$, while the two vertices of the tile that are opposite them are moved vertically down through distance $z$. The two remaining vertices are left in place. It is clear that the six points determined by this method form the vertices of an affinely regular hexagon. In Fig. 17 we show the arrangement of the six hexagons

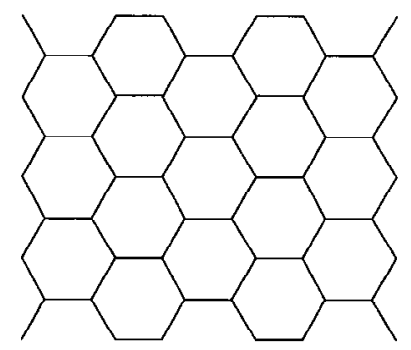

Fig. 16 


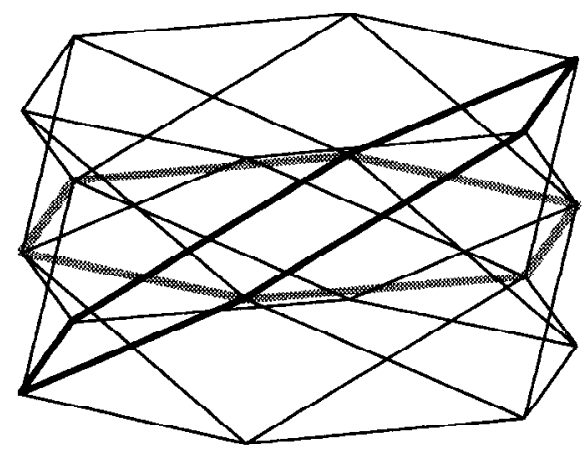

Fig. 17

arising from one hexagonal tile (which is shown by a grey line), for $z=0.5$. Our infinite polyhedron $P$ is the family of all such hexagons. It is, in fact, a slab-like structure, and each vertical line that goes through an interior point of the original tiling meets six faces of the "polyhedron" $P$. It is easy to verify that $P$ is orientable, isogonal, and isohedral. As in the previous cases, there is one exceptional situation: for $z=0$, the six hexagons generated from each hexagonal tile occupy the same position as the tile itself (but they are still distinguishable by their labels). It is easily verified that this "sixfold tiling" (or polyhedron) is, in fact, a regular tiling with overlapping tiles (see Chapter 12 of [11]), and with symbol (6.6.6.6.6.6) or, considered as a regular polyhedron, with the Schläfli symbol $\left\{6, \frac{6}{2}\right\}$.

\section{Some General Considerations}

The reader may be disappointed by the results of this paper, in that the most interesting conclusions do not deal with "real polyhedra" but with newfangled concepts masquerading under the name "polyhedra." In this last section I would like to show that these concept are both natural and convenient, and to explain why they have not been introduced earlier. Additional aspects of the theory of general polyhedra are discussed in [4]-[10].

In fact, the last part is easiest. There is in the literature no definition of general geometric polyhedra. Writers that deal with polyhedra that may have self-intersections either refrain from giving any definitions, or else, like Hess, Brückner, and most other early authors, give definitions to which they do not adhere. The problem actually started much earlier, with Poinsot in 1809. In his famous and widely quoted paper [15], Poinsot misinterpreted his own definition of regular polygons, and excluded by unjustified fiat regular polygons such as $\left\{\frac{6}{2}\right\}$ from any further consideration. This arbitrary exclusion has become universally accepted by all people investigating polyhedra (including the present writer till a few years ago). More details about the history of Poinsot's error, and about the rôle played by the historian Siegmund Günther in distorting the achievements of A. F. L. Meister (who long before Poinsot realized the utility of allowing polygons with coinciding vertices), can be found in [7]. It is rather remarkable that geometers 
(such as J. Douglas, B. H. Neumann, I. J. Schoenberg, F. Bachmann, and many others) investigating other aspects of the theory of polygons had no difficulty in following Meister and accepting $\left\{\frac{6}{2}\right\}$ and other polygons with coinciding vertices. In fact, the inclusion of these polygons is a prerequisite of the theories they developed. This topic will be discussed in detail in [13].

On the other hand, if vertices of polygons are not allowed to occupy the same points, it is natural that a variety of restrictions have to be imposed when considering what constitutes a polyhedron. The different directions of research then tended (and still tend) to impose conditions that make it easier to deal with the particular topic, without much concern about the needs of other topics. This accounts for restrictions such as: adjacent faces cannot be coplanar; the intersection of two faces must have dimension at most 1 ; two edges cannot have an intersection of positive length; and many others. Each of these restrictions is disregarded in other works, often by the same authors. The aim of the present note is to show by examples that it is as profitable in the theory of polyhedra to allow more freedom than traditionally granted, as it is in the theory of polygons.

One additional aspects of the topic of polyhedra adds to its complexity- the wish to have the totality of polyhedra closed under certain natural operations. First, it seems most desirable to define polyhedra and concepts related to them in such a way that they are preserved under continuous changes. In considering a quadratic equation it is advantageous to say that it always has two solutions, even though sometimes the two may have the same value. The same benefits accrue to the theory of polyhedra by preserving the identities of the vertices even though they may be represented by coinciding points. However, this has very weighty consequences. For example, if one restricts attention to convex polyhedra, then the facial structure (in the traditional understanding) is not invariant under continuous changes: limits of convex polyhedra of a certain type may fail to have the same type; even the numbers of faces (of different dimensions) are only lower semicontinuous [3]. Requiring continuity means that labels must be used to distinguish coinciding vertices, and that coplanarity of faces and collinearity of edges must be admitted. If limits of acoptic polyhedra (polyhedra without self-intersections) are to be included in the theory, overlapping faces and other elements need to be allowed, again enlarging the class. Thus, the definition presented and used here seems to be one that determines the smallest class closed under limits; this is why polyhedra as defined here should be considered a natural concept.

The second operation that is very desirable in the context of polyhedra is the transition from a polyhedron to a dual one. It is easily seen that the transition from a polyhedron to one obtained from it by polarity in a sphere is a duality, and it appears to be the only systematic way to obtain dual polyhedra; however, carrying out this transition is more intricate than commonly assumed [12]. Polarity requires that the position of the center of the reciprocating sphere not belong to any plane of a face. It requires that if nonconvex but acoptic polyhedra are included, then self-intersecting polyhedra also be included. The most fateful consequence of polarity, however, is the fact that if pairs of faces are allowed to have two or more edges in their intersection, then their polars will have pairs of vertices joined by several distinct edges. Such intersections are possible even with acoptic polyhedra, and they are very natural if polygons with coinciding vertices are admitted. For example, the regular polyhedron of type $\left\{\frac{8}{2}, 3\right\}$, presented in [7] and shown in Fig. 18(a), is a realization of the regular map shown in Fig. 18(b); each pair of 


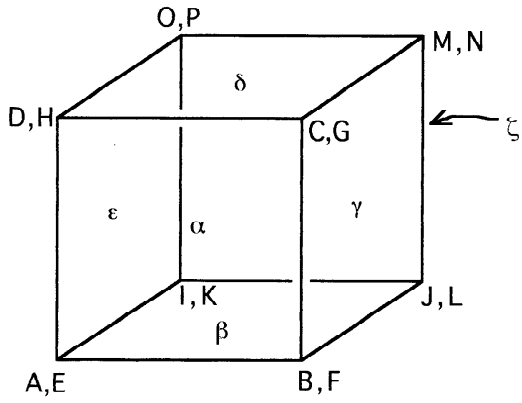

(a)

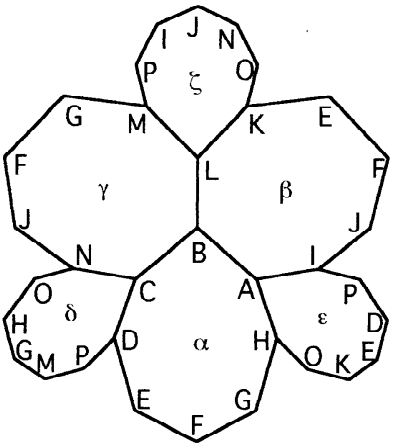

(b)

Fig. 18

faces that shares an edge share, in fact, two distinct edges. In any dual polyhedron, pairs of adjacent vertices would have to be joined by two distinct edges. While this is a wellestablished possibility in the theory of topological maps, it is beyond the capabilities of the polyhedral concept as presented here. A far-reaching generalization is required in which new labeling rules for vertices, edges, and faces need to be set up. This goes beyond the framework of the present note, and will be considered in a separate publication.

\section{References}

1. J. Bokowski and J. M. Wills, Regular polyhedra with hidden symmetries. Math. Intelligencer 10 (1988), 27-32.

2. U. Brehm and J. M. Wills, Polyhedral manifolds. In: Handbook of Convex Geometry, P. M. Gruber and J. M. Wills, eds. North-Holland, Amsterdam, 1993, pp. 535-554.

3. H. G. Eggleston, B. Grünbaum, and V. Klee, Some semicontinuity theorems for convex polytopes and cell complexes. Comment. Math. Helv. 39 (1964), 165-188.

4. B. Grünbaum, Regular polyhedra—old and new. Aequationes Math. 16 (1977), 1-20.

5. B. Grünbaum, Regularity of graphs, complexes and designs. Problèmes Combinatoires et Théorie des Graphes. Colloq. Internat. C.N.R.S. No. 260, Paris, 1978, pp. 191-197.

6. B. Grünbaum, Polyhedra with hollow faces. In: POLYTOPES: Abstract, Convex and Computational (Proc. NATO-ASI Conference, Toronto, 1993), T. Bisztriczky, P. McMullen, R. Schneider, and A. Ivic' Weiss, eds. Kluwer, Dordrecht, 1994, pp. 43-70.

7. B. Grünbaum, Regular polyhedra. In: Companion Encyclopedia of the History and Philosophy of the Mathematical Sciences, Vol. 2, I. Grattan-Guinness, ed. Routledge, London, 1994, pp. 866-876.

8. B. Grünbaum, Isogonal prismatoids. Discrete Comput. Geom. 18 (1997), 13-51.

9. B. Grünbaum, Acoptic polyhedra. Proc. Summer Research Conf. "Discrete and Computational Geometry: Ten Years Later" (to appear).

10. B. Grünbaum, Polyhedral realizations of a regular map of genus 5 (in preparation).

11. B. Grünbaum and G. C. Shephard, Tilings and Patterns. Freeman, New York, 1986.

12. B. Grünbaum and G. C. Shephard, Duality of polyhedra. In: Shaping Space: A Polyhedral Approach (Proc. "Shaping Space" Conference, Smith College, April 1984), M. Senechal and G. Fleck, eds. Birkhäuser, Boston, 1988, pp. 205-211.

13. B. Grünbaum and G. C. Shephard, Napoleon's theorem and its relatives (in preparation). 
14. P. McMullen, E. Schulte, and J. M. Wills, Infinite series of combinatorially regular polyhedra in threespace. Geom. Dedicata 26 (1988), 299-307.

15. L. Poinsot, Mémoire sur les polygones et les polyèdres. J. École Polytech. 4 (1810), 16-49. German translation, illustrations, and commentary by R. Haußner in: Abhandlungen über die regelmäßigen Sternkörper, Ostwald's Klassiker der exakten Wissenschaften, Nr. 151. Engelmann, Leipzig 1906, pp. 3-48

16. E. Schulte and J. M. Wills, A polyhedral realization of Felix Klein's map $\{3,7\}_{8}$ on a Riemann surface of genus 3. J. London Math. Soc. (2) 32 (1985), 539-547.

17. E. Schulte and J. M. Wills, Geometric realizations for Dyck's regular map on a surface of genus 3. Discrete Comput. Geom. 1 (1986), 141-153.

18. E. Schulte and J. M. Wills, On Coxeter's regular skew polyhedra. Discrete Math. 60 (1986), 253-262.

19. E. Schulte and J. M. Wills, Kepler-Poinsot-type realizations of regular maps of Klein, Fricke, Gordan and Sherk. Canad. Math. Bull. 30 (1987), 155-164.

20. E. Schulte and J. M. Wills, Combinatorially reagular polyhedra in three-space. In: Symmetry of Discrete Mathematical Structures and Their Symmetry Groups, K. H. Hofmann and R. Wille, eds. Heldermann Verlag, Berlin, 1990, pp. 49-88.

21. F. A. Sherk, A family of regular maps of type $\{6,6\}$. Canad. Math. Bull. 5 (1962), 13-20.

22. J. M. Wills, Reguläre polyedrische Mannigfaltigkeiten. Mitt. Math. Ges. Hamburg 11 (1983), 171-177.

23. J. M. Wills, Semi-Platonic manifolds. In: Convexity and Its Applications, P. M. Gruber and J. M. Wills, eds. Birkhäuser, Basel, 1983, 413-421.

24. J. M. Wills, On polyhedra with transitivity properties. Discrete Comput. Geom. 1 (1986), 195-199.

25. J. M. Wills, A combinatorially regular dodecahedron of genus 3. Discrete Math. 67 (1987), 199-204.

26. J. M. Wills, The combinatorially regular polyhedra of index 2. Aequationes Math. 34 (1987), 206-220.

27. J. M. Wills, On regular polyhedra with hidden symmetries. Results in Math. 12 (1987), 450-458.

28. J. M. Wills, Diskontinuierliche reguläre Polyeder. Rad. Jugoslav. Akad. 435 (1988), 65-73.

29. S. E. Wilson, New techniques for the construction of regular maps. Ph.D. thesis, University of Washington, Seattle, WA, 1976.

30. S. E. Wilson, Operators over regular maps. Pacific J. Math. 81 (1979), 559-568.

Received February 3, 1997, and in revised form August 5, 1997. 